\title{
Concentrations of Nutrients and Sedimert from Two Sites in the Spring Creek Basin, Benton County, Washington, 1997-98
}

By Richard J. Wagner

U.S. GEOLOGICAL SURVEY

Open-File Report 99-274

Prepared in cooperation with

BENTON CONSERVATION DISTRICT

Tacoma, Washington

2000 


\section{U.S. DEPARTMENT OF THE INTERIOR \\ BRUCE BABBITT, Secretary}

U.S. GEOLOGICAL SURVEY

Charles G. Groat, Director

The use of firm, trade, and brand names in this report is for identification purposes only and does not constitute endorsement by the U.S. Geological Survey

For additional information write to:

District Chief U.S. Geological Survey 1201 Pacific Avenue, Suite 600 Tacoma, Washington 98402
Copies of this report can be purchased from:

U.S. Geological Survey

Information Services

Box 25286

Federal Center

Denver, CO 80225 


\section{CONTENTS}

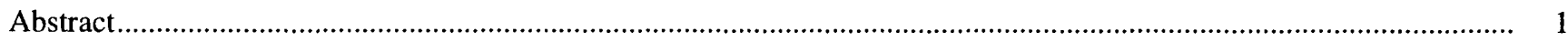

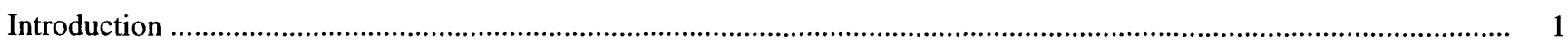

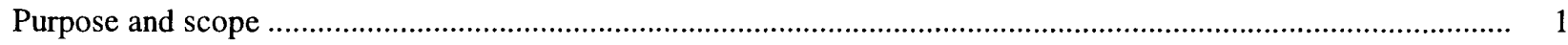

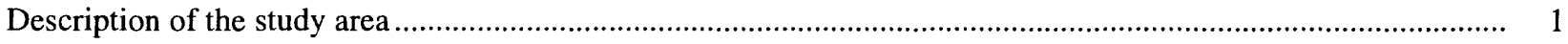

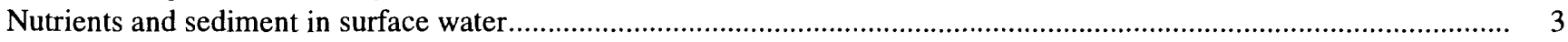

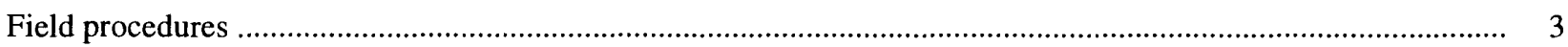

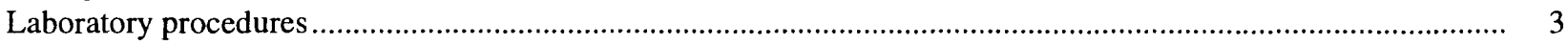

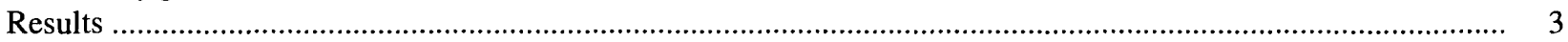

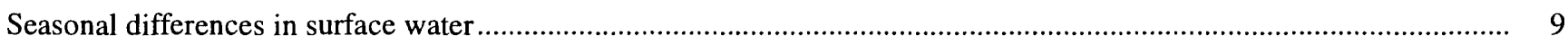

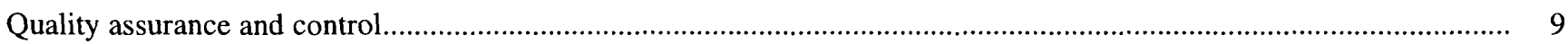

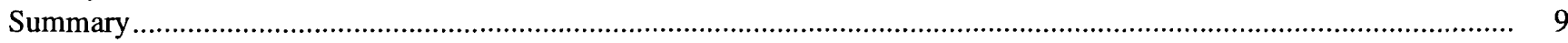

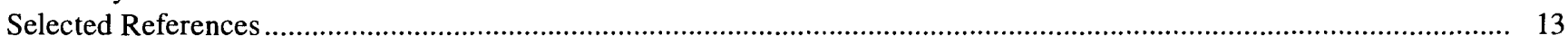

FIGURE

1. Locations of surface-water sites sampled for nutrients and suspended sediment in the Spring Creek Basin, Benton County, Washington

\section{TABLES}

1. Water discharge, values of temperature, specific conductance, dissolved oxygen, $\mathrm{pH}$, and concentrations of nutrients and suspended sediment in samples collected April 1997 to September 1998 in the Spring Creek Basin, Benton, County, Washington.

2. Statistical summary of selected water-quality data collected from April 1997 to September 1998 in the Spring Creek Basin, Benton County, Washington .

3. Nutrient and sediment loads from two sites in the Spring Creek Basin, April 1997 to September 1998, Benton County, Washington

\section{CONVERSION FACTORS AND VERTICAL DATUM}

\begin{tabular}{lcl} 
Multiply & By & To obtain \\
\hline microgram $(\mu \mathrm{g})$ & $3.53 \times 10^{-8}$ & ounce \\
micrometer $(\mu \mathrm{m})$ & $3.937 \times 10^{-5}$ & inch \\
liter $(\mathrm{L})$ & 0.296 & gallon \\
tons per day $(\mathrm{t} / \mathrm{day})$ & 910.72 & kilogram per day \\
square mile $\left(\mathrm{mi}^{2}\right)$ & 2.590 & square kilometer
\end{tabular}

Temperature: To convert temperature given in this report in degrees Fahrenheit $\left({ }^{\circ} \mathrm{F}\right)$ to degrees Celsius $\left({ }^{\circ} \mathrm{C}\right)$, use the following equation: ${ }^{\circ} \mathrm{C}=5 / 9\left({ }^{\circ} \mathrm{F}-32\right)$.

Sea Level: In this report "sea level" refers to the National Geodetic Vertical Datum of 1929 (NGVD of 1929)-a geodetic datum derived from a general adjustment of the first-order level nets of both the United States and Canada, formerly called Sea Level Datum of 1929. 


\title{
Concentrations of Nutrients and Sediment from Two Sites in the Spring Creek Basin, Benton County, Washington, 1997-98
}

\author{
Richard J. Wagner
}

\section{ABSTRACT}

From April 1997 to September 1998, the U.S. Geological Survey sampled streamflow from two sites in the Spring Creek Basin. Eighteen samples were collected from Spring Creek at Hanks Road and nineteen from Spring Creek at McCreadie Road and analyzed for concentrations of nitrogen, phosphorus, and sediment. Measurements of temperature, $\mathrm{pH}$, specific conductance, alkalinity, and dissolved oxygen also were made at the time of collection of nutrient and sediment samples. This report presents physical properties and chemical data collected during the study period from Spring Creek at Hanks Road and Spring Creek at McCreadie Road.

\section{INTRODUCTION}

The Benton Conservation District (BCD), under the Washington Department of Ecology Centennial Clean Water Program, has a long-term goal to reduce the amount of sediment entering the Yakima River through irrigation runoff to its major tributaries in Benton County. To help determine if efforts to reduce sediment yields are effective, the BCD entered into a cooperative agreement with the U.S. Geological Survey (USGS) to install a gage to measure streamflow in Spring Creek and collect surface-water samples for the analysis of nitrogen and phosphorus (nutrients) from two sites on Spring Creek, April 1997 through
September 1998. These data will provide baseline information about nutrient concentrations in surface water in the Spring Creek watershed. Measurements of temperature, $\mathrm{pH}$, specific conductance, alkalinity, and dissolved oxygen also were made at the time of collection of nutrient and sediment samples.

\section{Purpose and Scope}

The purpose of this report is to present data for the concentrations and loads of nutrients and se liment in surface water from two sites on Spring Creel from April 1997 to September 1998. Streamflow data are published in Wiggins and others $(1998,1999)$.

\section{Description of the Study Area}

Spring Creek is a major tributary of the lower reach of the Yakima River in Benton County (fig. 1) and has a drainage area of approximately $32 \mathrm{mi}^{2}$ (square miles) (Daly, 1998). The creek provides a source of irrigation water and receives irrigation runoff from fields and drains in the Spring Creek watershed. Irrigation in the Spring Creek Basin is primarily by sprinkler and rill methods, although there is a small amount of drip irrigation. Primary crops growr in the study area are apples, wine and table grapes, hons, and a smaller percentage of cherries and other fruits (Daly, 1998). 


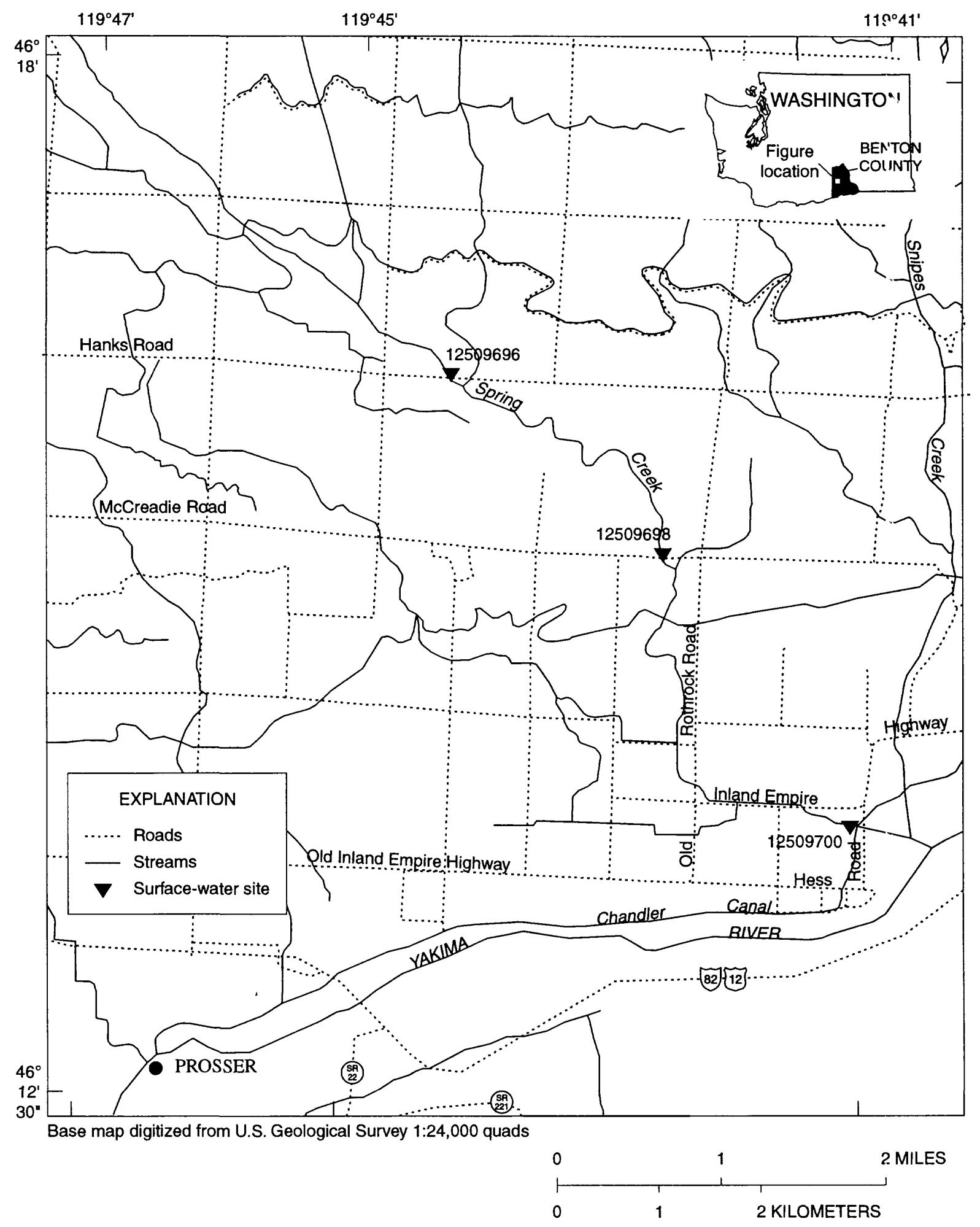

Figure 1. Locations of surface-water sites sampled for nutrients and suspended sediment in the Spring Creek Basin, Benton County, Washington. 


\section{NUTRIENTS AND SEDIMENT IN SURFACE WATER}

Two sites on Spring Creek, at Hanks Road and at McCreadie Road (fig. 1), were selected as being representative of land-use conditions for sampling the Spring Creek watershed upstream from the respective sites of collection. Eighteen samples were collected from the Hanks Road site and 19 samples from the McCreadie Road site from April 1997 through September 1998. Samples were analyzed for nitrogen and phosphorus species (nutrients), suspended sediment, and physical measurements were made at the sites to determine temperature, dissolved oxygen, $\mathrm{pH}$, alkalinity, streamflow, and specific conductance.

\section{Field Procedures}

Water samples representative of the stream were obtained by collecting depth-integrated subsamples at equally spaced verticals across the stream using a US DH-81 sampler, as described by Edwards and Glysson (1988). Subsamples were composited in a polyethylene churn and either split for raw-water samples (unfiltered) or filtered through a 0.45 -micrometer $(\mu \mathrm{m})$ filter, as described by Horowitz and others (1994). Samples for the analysis of nutrients were stored at less than 4 degrees Celsius $\left({ }^{\circ} \mathrm{C}\right)$ and submitted to the U.S. Geological Survey National Water Quality Laboratory (NWQL) in Arvada, Colo., for analysis. Samples for the analysis of suspended sediment were collected in conjunction with nutrient samples but were not composited in the churn splitter. Suspended sediment samples were submitted to the USGS Cascades Volcano Observatory Sediment Laboratory in Vancouver, Wash., for analysis as described by Guy (1969).

\section{Laboratory Procedures}

Water samples for the analysis of nutrients were received at the NWQL and stored at less than $4^{\circ} \mathrm{C}$ prior to analysis, as described by Pritt and Raese (1995).

Samples were analyzed for nitrite using a diazotization colorimetric method, ammonia using a salicylatehypochlorite colorimetric method, nitrate-plus-nitrite using cadmium reduction-diazotization colorimetric method, and orthophosphate using a phosphomolybdate colorimetric method; all as described by Fishman (1993). Samples were analyzed for ammonia- plus-organic nitrogen as well as phosphorus using microkjeldahl digestion and colorimetry, as des ribed by Patton and Truitt (1992). Where applicable, dissolved concentrations were measured using samples filtered through a 0.45 -micrometer filter and total concentrations (or whole-water samples) were measured using unfiltered samples.

\section{Results}

All data collected are shown in table 1. The majority of nitrite concentrations at both sites was less than the detection limit of 0.01 milligrams per liter $(\mathrm{mg} / \mathrm{L})($ table 1$)$ and the maximum nitrite value observed was $0.02 \mathrm{mg} / \mathrm{L}$ (table 2). Because of these low values for nitrite, this report will hereafter refer to nitrate-plus-nitrite simply as nitrate. Observed concentrations of nitrate in samples from Spring Creek at Hanks Road ranged from 0.37 to $6.1 \mathrm{mg} / \mathrm{L}$, with a median of $1.2 \mathrm{mg} / \mathrm{L}$ (table 2 ); whereas concentrations in samples from the McCreadie Road site range $\mathrm{I}$ from 0.94 to $6.9 \mathrm{mg} / \mathrm{L}$ with a median of $2.0 \mathrm{mg} / \mathrm{L}$. This compares with a median value of $1.4 \mathrm{mg} / \mathrm{L}$ for samples collected from Spring Creek at Hess Road (see fig. 1) during 1974-81 (Rinella and others, 1992). Concentrations of filtered phosphorus in samples from Spring Creek at Hanks Road ranged from 0.01 to $0.07 \mathrm{mg} / \mathrm{L}$, with a median of $0.04 \mathrm{mg} / \mathrm{L}$; and concentrations in samples from the McCreadie Road site ranged from 0.02 to $0.11 \mathrm{mg} / \mathrm{L}$, with an identical median of $0.04 \mathrm{mg} / \mathrm{L}$. Concentrations of total phosphorus in samples from Spring Creek at Hanks Road ranged from 0.05 to $0.26 \mathrm{mg} / \mathrm{L}$ with a median of $0.09 \mathrm{mg} / \mathrm{L}$; and concentrations in samples from the McCreadie Road site ranged from 0.03 to 0.54 , with a median of $0.11 \mathrm{mg} / \mathrm{L}$. Concentrations of suspended sediment in samples from Spring Creek at Hanks Road ranged from 4 to $226 \mathrm{mg} / \mathrm{L}$, with a median of $42 \mathrm{mg} / \mathrm{L}$; comp?red to concentrations from the McCreadie Road site tl at ranged from 6 to $664 \mathrm{mg} / \mathrm{L}$, with a median of $8 \varepsilon \mathrm{mg} / \mathrm{L}$. 
Table 1. Water discharge, values of temperature, specific conductance, dissolved oxygen, $\mathrm{pH}$, and concentrations of nutrients and suspended sediment in samples collected April 1997 to September 1998 in the Spring Creek Basin, Benton County, Washington

$\left[{ }^{\circ} \mathrm{C}\right.$, degrees Celsius; $\mu \mathrm{S} / \mathrm{cm}$, microsiemens per centimeter; $\mathrm{mg} / \mathrm{L}$, milligrams per liter; $\mathrm{N}$, nitrogen; ammonia + organic, ammonia plus organic; $\mathrm{NO}_{2}+\mathrm{NO}_{3}$, nitrite plus nitrate; $\mathrm{P}$, phosphorus; $\mathrm{t} / \mathrm{day}$, tons per day; <, less than; ,-- not available]

12509696--Spring Creek at Hanks Road near Prosser, Washington

\begin{tabular}{|c|c|c|c|c|c|c|c|c|c|}
\hline Date & Time & $\begin{array}{l}\text { Temper- } \\
\text { ature } \\
\text { water } \\
\left({ }^{\circ} \mathrm{C}\right)\end{array}$ & $\begin{array}{l}\text { Dis- } \\
\text { charge, } \\
\text { inst. } \\
\text { (cubic } \\
\text { feet } \\
\text { per } \\
\text { second) }\end{array}$ & $\begin{array}{l}\text { Spe- } \\
\text { cific } \\
\text { con- } \\
\text { duct- } \\
\text { ance } \\
(\mu \mathrm{S} / \mathrm{cm})\end{array}$ & $\begin{array}{l}\text { Oxygen, } \\
\text { dis- } \\
\text { solved } \\
(\mathrm{mg} / \mathrm{L})\end{array}$ & $\begin{array}{l}\text { Oxygen, } \\
\text { dis- } \\
\text { solved } \\
\text { (per- } \\
\text { cent } \\
\text { satur- } \\
\text { ation) }\end{array}$ & $\begin{array}{l}\mathrm{pH} \\
\text { water } \\
\text { whole } \\
\text { field } \\
\text { (stand- } \\
\text { ard } \\
\text { units) }\end{array}$ & $\begin{array}{l}\text { Nitro- } \\
\text { gen, } \\
\text { ammonia } \\
\text { dis- } \\
\text { solved } \\
(\mathrm{mg} / \mathrm{L} \\
\text { as } \mathrm{N})\end{array}$ & $\begin{array}{l}\text { Nitro- } \\
\text { gen, } \\
\text { nitrite } \\
\text { dis- } \\
\text { solved } \\
\text { (mg/L } \\
\text { as } \mathrm{N})\end{array}$ \\
\hline \multicolumn{10}{|c|}{ April 1997} \\
\hline $\begin{array}{l}23 \ldots \\
\text { May }\end{array}$ & \multicolumn{6}{|c|}{ May } & & & $<0.01$ \\
\hline $\begin{array}{c}23 \ldots \\
\text { June }\end{array}$ & \multicolumn{8}{|c|}{ June } & 0.01 \\
\hline $\begin{array}{c}16 \ldots \\
\text { July }\end{array}$ & 1315 & 20.0 & 7.7 & 230 & 8.3 & 94 & 7.9 & 0.02 & 0.02 \\
\hline $\begin{array}{c}16 \ldots \\
\text { August }\end{array}$ & 1200 & 18.5 & 9.3 & 185 & 8.0 & 88 & 7.8 & $<0.015$ & $<0.01$ \\
\hline $\begin{array}{r}20 \ldots \\
\text { Septem }\end{array}$ & 1310 & 18.5 & 9.7 & 216 & 6.7 & 74 & 7.7 & 0.04 & 0.01 \\
\hline $\begin{array}{c}24 \ldots \\
\text { October }\end{array}$ & 1400 & 17.0 & 7.2 & 269 & 7.7 & 82 & 7.8 & $<0.015$ & $<0.01$ \\
\hline $\begin{array}{c}22 \ldots \\
\text { Novemb }\end{array}$ & 1300 & 11.5 & 3.2 & 454 & 10.2 & 97 & 7.8 & $<0.015$ & 0.01 \\
\hline $\begin{array}{c}25 \ldots \\
\text { Decemb }\end{array}$ & 1330 & 10.5 & 1.4 & 640 & 9.8 & 90 & 8.0 & $<0.02$ & $<0.01$ \\
\hline $\begin{array}{c}18 \ldots \\
\text { January }\end{array}$ & $8^{1300}$ & 7.5 & 0.80 & 660 & 10.8 & 92 & 7.8 & $<0.02$ & $<0.01$ \\
\hline $\begin{array}{c}27 \ldots \\
\text { Februar }\end{array}$ & 1245 & 9.0 & 0.39 & 670 & 11.2 & 100 & 8.0 & $<0.02$ & 0.01 \\
\hline $\begin{array}{r}25 \ldots \\
\text { March }\end{array}$ & 1245 & 8.0 & 0.40 & 670 & 12.0 & 105 & 8.3 & $<0.02$ & 0.02 \\
\hline $\begin{array}{c}18 \ldots \\
\text { April }\end{array}$ & 1230 & 11.0 & 0.15 & 650 & 13.2 & 123 & 8.4 & 0.02 & 0.01 \\
\hline $\begin{array}{l}23 \ldots \\
\text { May }\end{array}$ & 1330 & 16.5 & 4.1 & 181 & 9.0 & 96 & 8.2 & 0.04 & $<0.01$ \\
\hline $\begin{array}{c}19 \ldots \\
\text { June }\end{array}$ & 1240 & 16.0 & 7.4 & 178 & 9.6 & 101 & 8.0 & 0.04 & $<0.01$ \\
\hline $\begin{array}{l}24 \ldots \\
\text { July }\end{array}$ & 1300 & 17.0 & 5.4 & 250 & 8.4 & 91 & 7.9 & 0.04 & $<0.01$ \\
\hline $\begin{array}{c}22 \ldots \\
\text { August }\end{array}$ & 1255 & 22.5 & 7.8 & 156 & 7.7 & 92 & 7.7 & 0.03 & $<0.01$ \\
\hline $\begin{array}{c}20 \ldots \\
\text { Septeml }\end{array}$ & 1240 & 20.0 & 11 & 175 & 7.7 & 88 & 7.6 & 0.07 & 0.01 \\
\hline $08 \ldots$ & 1200 & 19.5 & 11 & 204 & 7.5 & 85 & 7.8 & 0.04 & $<0.01$ \\
\hline
\end{tabular}


Table 1. Water discharge, values of temperature, specific conductance, dissolved oxygen, $\mathrm{pH}$, and concentrations of nutrients and suspended sediment in samples collected April 1997 to September 1998 in the Spring Creek Basin,

Benton County, Washington--Continued

12509696--Spring Creek at Hanks Road near Prosser, Washington

\begin{tabular}{|c|c|c|c|c|c|c|c|c|c|}
\hline Date & $\begin{array}{l}\text { Nitro- } \\
\text { gen, am- } \\
\text { monia + } \\
\text { organic } \\
\text { dis. } \\
(\mathrm{mg} / \mathrm{L} \\
\text { as } \mathrm{N})\end{array}$ & $\begin{array}{l}\text { Nitro- } \\
\text { gen, am- } \\
\text { monia + } \\
\text { organic } \\
\text { total } \\
(\mathrm{mg} / \mathrm{L} \\
\text { as } \mathrm{N})\end{array}$ & $\begin{array}{l}\text { Nitro- } \\
\text { gen, } \\
\mathrm{NO}_{2}+\mathrm{NO}_{3} \\
\text { dis- } \\
\text { solved } \\
(\mathrm{mg} / \mathrm{L} \\
\text { as } \mathrm{N})\end{array}$ & $\begin{array}{l}\text { Nitro- } \\
\text { gen, } \\
\text { total } \\
(\mathrm{mg} / \mathrm{L} \\
\text { as } \mathrm{N})\end{array}$ & $\begin{array}{l}\text { Phos- } \\
\text { phorus } \\
\text { total } \\
(\mathrm{mg} / \mathrm{L} \\
\text { as } \mathrm{P} \text { ) }\end{array}$ & $\begin{array}{l}\text { Phos- } \\
\text { phorus } \\
\text { dis- } \\
\text { solved } \\
\text { (mg/L } \\
\text { as P) }\end{array}$ & $\begin{array}{l}\text { Phos- } \\
\text { phorus } \\
\text { ortho, } \\
\text { dis- } \\
\text { solved } \\
\text { (mg/L } \\
\text { as } \mathrm{P} \text { ) }\end{array}$ & $\begin{array}{l}\text { Sedi- } \\
\text { ment, } \\
\text { sus- } \\
\text { pended } \\
(\mathrm{mg} / \mathrm{L})\end{array}$ & $\begin{array}{l}\text { Sedi- } \\
\text { ment, } \\
\text { dis- } \\
\text { charge, } \\
\text { sus- } \\
\text { pended } \\
\text { (t/day) }\end{array}$ \\
\hline \multicolumn{10}{|c|}{ April 1997} \\
\hline $\begin{array}{c}23 \ldots \\
\text { May }\end{array}$ & \multicolumn{8}{|c|}{ May } & 1.7 \\
\hline $\begin{array}{r}23 \ldots \\
\text { June }\end{array}$ & 0.2 & 0.6 & 1.5 & 2.1 & 0.23 & 0.04 & 0.04 & 226 & 2.7 \\
\hline $\begin{array}{c}16 \ldots \\
\text { July }\end{array}$ & $<0.2$ & 0.6 & 1.1 & 1.7 & 0.26 & 0.05 & 0.05 & 198 & 4.1 \\
\hline $\begin{array}{c}16 \ldots \\
\text { August }\end{array}$ & $<0.2$ & 0.2 & 0.82 & 1.1 & 0.10 & 0.01 & 0.03 & 38 & 0.96 \\
\hline \multicolumn{10}{|c|}{ September } \\
\hline $\begin{array}{c}24 \ldots \\
\text { Octobe }\end{array}$ & $<0.2$ & $<0.2$ & 1.3 & -- & 0.05 & 0.02 & 0.03 & 12 & 0.23 \\
\hline $\begin{array}{r}22 \ldots \\
\text { Novem }\end{array}$ & $<0.2$ & $<0.2$ & 3.2 & -- & 0.06 & 0.03 & 0.04 & 22 & 0.19 \\
\hline $\begin{array}{r}25 \ldots \\
\text { Decem }\end{array}$ & \multicolumn{9}{|c|}{ December } \\
\hline $\begin{array}{c}18 \ldots \\
\text { January }\end{array}$ & \multicolumn{8}{|c|}{ January 19} & 0.10 \\
\hline $\begin{array}{c}27 \ldots \\
\text { Februar }\end{array}$ & 0.2 & 0.3 & 6.1 & 6.4 & 0.05 & 0.04 & 0.07 & 4 & 0.00 \\
\hline $\begin{array}{r}25 \ldots \\
\text { March }\end{array}$ & 0.3 & 0.3 & 5.6 & 6.0 & 0.07 & 0.07 & 0.07 & 135 & 0.15 \\
\hline $\begin{array}{c}18 \ldots \\
\text { April }\end{array}$ & 0.1 & 0.3 & 5.3 & 5.6 & 0.06 & 0.05 & 0.05 & 14 & 0.01 \\
\hline $\begin{array}{l}23 \ldots \\
\text { May }\end{array}$ & \multicolumn{8}{|c|}{ May } & 0.80 \\
\hline $\begin{array}{c}19 \ldots \\
\text { June }\end{array}$ & 0.2 & 0.3 & 0.61 & 0.92 & 0.09 & 0.03 & 0.03 & 112 & 2.2 \\
\hline $\begin{array}{l}24 \ldots \\
\text { July }\end{array}$ & 0.2 & 0.4 & 0.90 & 1.3 & 0.09 & 0.04 & 0.04 & 46 & 0.67 \\
\hline $\begin{array}{c}22 \ldots \\
\text { August }\end{array}$ & 0.2 & 0.3 & 0.37 & 0.65 & 0.12 & 0.04 & 0.03 & 28 & 0.59 \\
\hline $\begin{array}{r}20 \ldots \\
\text { Septem }\end{array}$ & 0.2 & 0.2 & 0.43 & 0.66 & 0.05 & 0.03 & 0.05 & 11 & 0.32 \\
\hline $08 \ldots$ & 0.2 & 0.3 & 0.56 & 0.86 & 0.10 & 0.05 & 0.06 & 28 & 0.85 \\
\hline
\end{tabular}


Table 1. Water discharge, values of temperature, specific conductance, dissolved oxygen, $\mathrm{pH}$, and concentrations of nutrients and suspended sediment in samples collected April 1997 to September 1998 in the Spring Creek Basin, Benton County, Washington--Continued

12509698--Spring Creek at McCreadie Road near Prosser, Washington

\begin{tabular}{|c|c|c|c|c|c|c|c|c|c|}
\hline Date & Time & $\begin{array}{l}\text { Temper- } \\
\text { ature } \\
\text { water } \\
\left({ }^{\circ} \mathrm{C}\right)\end{array}$ & $\begin{array}{l}\text { Dis- } \\
\text { charge, } \\
\text { inst. } \\
\text { (cubic } \\
\text { feet } \\
\text { per } \\
\text { second) }\end{array}$ & $\begin{array}{l}\text { Spe- } \\
\text { cific } \\
\text { con- } \\
\text { duct- } \\
\text { ance } \\
(\mu \mathrm{S} / \mathrm{cm})\end{array}$ & $\begin{array}{l}\text { Oxygen, } \\
\text { dis- } \\
\text { solved } \\
(\mathrm{mg} / \mathrm{L})\end{array}$ & $\begin{array}{l}\text { Oxygen, } \\
\text { dis- } \\
\text { solved } \\
\text { (per- } \\
\text { cent } \\
\text { satur- } \\
\text { ation) }\end{array}$ & $\begin{array}{l}\mathrm{pH} \\
\text { water } \\
\text { whole } \\
\text { field } \\
\text { (stand- } \\
\text { ard } \\
\text { units) }\end{array}$ & $\begin{array}{l}\text { Nitro- } \\
\text { gen, } \\
\text { ammonia } \\
\text { dis- } \\
\text { solved } \\
(\mathrm{mg} / \mathrm{L} \\
\text { as } \mathrm{N})\end{array}$ & $\begin{array}{l}\text { Nitro- } \\
\text { gen, } \\
\text { nitrite } \\
\text { dis- } \\
\text { solved } \\
\text { (mg/L } \\
\text { as } \mathrm{N} \text { ) }\end{array}$ \\
\hline \multicolumn{10}{|c|}{ April 1997} \\
\hline $23 \ldots$ & 1030 & 12.0 & 11 & 320 & 10.6 & 101 & 8.2 & $<0.015$ & $<0.01$ \\
\hline \multicolumn{10}{|l|}{ May } \\
\hline $\begin{array}{c}23 \ldots \\
\text { June }\end{array}$ & 1020 & 14.0 & 12 & 316 & 10.0 & 98 & 8.0 & $<0.015$ & $<0.01$ \\
\hline $\begin{array}{c}16 \ldots \\
\text { July }\end{array}$ & 1100 & 18.5 & 11 & 317 & 8.8 & 96 & 8.0 & $<0.015$ & 0.01 \\
\hline $\begin{array}{c}16 \ldots \\
\text { August }\end{array}$ & 0950 & 16.0 & 12 & 327 & 9.1 & 94 & 7.9 & $<0.015$ & $<0.01$ \\
\hline $\begin{array}{c}20 \ldots \\
\text { Septemb }\end{array}$ & 1000 & 17.5 & 14 & 315 & 8.6 & 93 & 7.9 & $<0.015$ & $<0.01$ \\
\hline $\begin{array}{c}24 \ldots \\
\text { October }\end{array}$ & 1205 & 16.0 & 16 & 380 & 9.2 & 96 & 8.0 & $<0.015$ & $<0.01$ \\
\hline $\begin{array}{c}22 \ldots \\
\text { Novemb }\end{array}$ & 1100 & 10.5 & 7.6 & 567 & 10.5 & 96 & 8.1 & $<0.015$ & $<0.01$ \\
\hline $\begin{array}{c}25 \ldots \\
\text { Decemb }\end{array}$ & 1050 & 10.5 & 4.2 & 738 & 10.6 & 97 & 8.2 & $<0.02$ & $<0.01$ \\
\hline $\begin{array}{c}18 \ldots \\
\text { January }\end{array}$ & $8^{1150}$ & 9.0 & 3.3 & 750 & 11.4 & 100 & 8.2 & $<0.02$ & $<0.01$ \\
\hline $\begin{array}{c}27 \ldots \\
\text { February }\end{array}$ & 1030 & 10.5 & 2.8 & 743 & 11.0 & 101 & 8.2 & $<0.02$ & $<0.01$ \\
\hline $\begin{array}{r}25 \ldots \\
\text { March }\end{array}$ & 1030 & 10.0 & 2.6 & 730 & 11.6 & 106 & 8.3 & $<0.02$ & $<0.01$ \\
\hline $\begin{array}{c}18 \ldots \\
\text { April }\end{array}$ & 1020 & 11.0 & 2.2 & 730 & 11.4 & 105 & 8.4 & 0.03 & $<0.01$ \\
\hline $\begin{array}{c}23 . . \\
\text { May }\end{array}$ & 1030 & 15.0 & 9.7 & 293 & 10.5 & 108 & 8.2 & 0.03 & $<0.01$ \\
\hline $\begin{array}{c}19 \ldots \\
\text { June }\end{array}$ & 1015 & 14.5 & 16 & 251 & 10.0 & 101 & 8.1 & 0.03 & $<0.01$ \\
\hline $\begin{array}{l}01 \ldots \\
24 \ldots\end{array}$ & $\begin{array}{l}1200 \\
1100\end{array}$ & $\begin{array}{l}18.5 \\
17.5\end{array}$ & $\begin{array}{l}17 \\
13\end{array}$ & $\begin{array}{l}236 \\
302\end{array}$ & $\begin{array}{l}9.6 \\
8.6\end{array}$ & $\begin{array}{r}106 \\
93\end{array}$ & $\begin{array}{l}8.0 \\
8.1\end{array}$ & $\begin{array}{l}0.08 \\
0.03\end{array}$ & $\begin{array}{r}0.02 \\
<0.01\end{array}$ \\
\hline July & & & & & & & & & \\
\hline $\begin{array}{r}22 \ldots \\
\text { August }\end{array}$ & 1040 & 20.5 & 15 & 260 & 8.3 & 95 & 7.9 & 0.03 & $<0.01$ \\
\hline $\begin{array}{c}20 \ldots \\
\text { Septemb }\end{array}$ & 1020 & 18.0 & 16 & 268 & 9.2 & 100 & 8.1 & 0.07 & $<0.01$ \\
\hline $08 \ldots$ & 1050 & 18.5 & 19 & 278 & 9.0 & 99 & 8.0 & 0.03 & $<0.01$ \\
\hline
\end{tabular}


Table 1. Water discharge, values of temperature, specific conductance, dissolved oxygen, $\mathrm{pH}$, and concentrations of nutrients and suspended sediment in samples collected April 1997 to September 1998 in the Spring Creek Basin, Benton County, Washington--Continued

\begin{tabular}{|c|c|c|c|c|c|c|c|c|c|}
\hline \multirow[b]{2}{*}{ Date } & \multirow[b]{2}{*}{$\begin{array}{l}\text { Nitro- } \\
\text { gen, am- } \\
\text { monia + } \\
\text { organic } \\
\text { dis. } \\
(\mathrm{mg} / \mathrm{L} \\
\text { as } \mathrm{N})\end{array}$} & \multicolumn{6}{|c|}{ 12509698--Spring Creek at McCreadie Road near Prosser, Washington } & \multirow[b]{2}{*}{$\begin{array}{l}\text { Sedi- } \\
\text { ment, } \\
\text { sus- } \\
\text { pended } \\
(\mathrm{mg} / \mathrm{L})\end{array}$} & \multirow[b]{2}{*}{$\begin{array}{l}\text { Sodi- } \\
\text { ment, } \\
\text { dis- } \\
\text { charge, } \\
\text { sus- } \\
\text { pended } \\
\text { (t/day) }\end{array}$} \\
\hline & & $\begin{array}{l}\text { Nitro- } \\
\text { gen, am- } \\
\text { monia + } \\
\text { organic } \\
\text { total } \\
(\mathrm{mg} / \mathrm{L} \\
\text { as } \mathrm{N})\end{array}$ & $\begin{array}{l}\text { Nitro- } \\
\text { gen, } \\
\mathrm{NO}_{2}+\mathrm{NO}_{3} \\
\text { dis- } \\
\text { solved } \\
\text { (mg/L } \\
\text { as } \mathrm{N})\end{array}$ & $\begin{array}{l}\text { Nitro- } \\
\text { gen, } \\
\text { total } \\
(\mathrm{mg} / \mathrm{L} \\
\text { as } \mathrm{N})\end{array}$ & $\begin{array}{l}\text { Phos- } \\
\text { phorus } \\
\text { total } \\
(\mathrm{mg} / \mathrm{L} \\
\text { as } \mathrm{P})\end{array}$ & $\begin{array}{l}\text { Phos- } \\
\text { phorus } \\
\text { dis- } \\
\text { solved } \\
\text { (mg/L } \\
\text { as P) }\end{array}$ & $\begin{array}{l}\text { Phos- } \\
\text { phorus } \\
\text { ortho, } \\
\text { dis- } \\
\text { solved } \\
(\mathrm{mg} / \mathrm{L} \\
\text { as } \mathrm{P})\end{array}$ & & \\
\hline \multicolumn{10}{|c|}{ April 1997} \\
\hline \multicolumn{10}{|l|}{ May } \\
\hline $\begin{array}{l}23 \ldots \\
\text { June }\end{array}$ & $<0.2$ & 0.6 & 2.2 & 2.7 & 0.23 & 0.04 & 0.04 & 328 & 10 \\
\hline $\begin{array}{l}16 \ldots \\
\text { July }\end{array}$ & $<0.2$ & 1.1 & 2.0 & 3.2 & 0.54 & 0.06 & 0.08 & 223 & 6.9 \\
\hline $\begin{array}{c}16 \ldots \\
\text { August }\end{array}$ & $<0.2$ & 0.3 & 2.0 & 2.3 & 0.10 & 0.02 & 0.03 & 88 & 2.9 \\
\hline $\begin{array}{c}20 \ldots \\
\text { Septemb }\end{array}$ & $<0.2$ & 0.3 & 1.7 & 2.0 & 0.08 & 0.05 & 0.05 & 71 & 2.7 \\
\hline \multicolumn{10}{|l|}{ October } \\
\hline $\begin{array}{c}22 \ldots \\
\text { Novemb }\end{array}$ & $<0.2$ & $<0.2$ & 4.2 & -- & 0.07 & 0.04 & 0.05 & 32 & 0.66 \\
\hline $\begin{array}{c}25 \ldots \\
\text { Decemb }\end{array}$ & 0.2 & 0.3 & 6.9 & 7.1 & 0.04 & 0.04 & 0.11 & 8 & 0.09 \\
\hline $\begin{array}{c}18 \ldots \\
\text { January }\end{array}$ & 0.2 & 0.3 & 6.8 & 7.0 & 0.08 & 0.06 & 0.12 & 6 & 0.05 \\
\hline \multicolumn{9}{|c|}{ February } & 0.05 \\
\hline \multicolumn{9}{|l|}{ March } & 0.62 \\
\hline $18 \ldots$ & 0.2 & 0.3 & 6.0 & 6.3 & 0.03 & 0.02 & 0.03 & 35 & 0.21 \\
\hline \multicolumn{9}{|l|}{ April } & 1.9 \\
\hline $\begin{array}{c}\text { May } \\
19 \ldots\end{array}$ & 0.1 & 0.3 & 1.2 & 1.6 & 0.11 & 0.03 & 0.05 & 172 & 7.3 \\
\hline \multicolumn{10}{|l|}{ June } \\
\hline $\begin{array}{l}01 \ldots \\
24 \ldots\end{array}$ & $\begin{array}{l}0.2 \\
0.2\end{array}$ & $\begin{array}{l}0.5 \\
0.4\end{array}$ & $\begin{array}{l}0.94 \\
1.5\end{array}$ & $\begin{array}{l}1.4 \\
1.9\end{array}$ & $\begin{array}{l}0.20 \\
0.11\end{array}$ & $\begin{array}{l}0.03 \\
0.05\end{array}$ & $\begin{array}{l}0.05 \\
0.06\end{array}$ & $\begin{array}{r}156 \\
78\end{array}$ & $\begin{array}{l}7.2 \\
2.7\end{array}$ \\
\hline \multicolumn{10}{|l|}{ July } \\
\hline $\begin{array}{c}22 \ldots \\
\text { August }\end{array}$ & 0.3 & 0.8 & 1.2 & 2.0 & 0.38 & 0.06 & 0.07 & 664 & 28 \\
\hline $\begin{array}{c}20 \ldots \\
\text { Septemb }\end{array}$ & 0.2 & 0.7 & 1.3 & 2.0 & 0.15 & 0.05 & 0.16 & 77 & 3.3 \\
\hline $08 \ldots$ & 0.2 & 0.4 & 1.3 & 1.8 & 0.20 & 0.08 & 0.07 & 130 & 6.6 \\
\hline
\end{tabular}


Table 2. Statistical summary of selected water-quality data collected from April 1997 to September 1998 in the Spring Creek Basin, Benton County, Washington

$\left[\%\right.$, percent; degrees $\mathrm{C}\left({ }^{\circ} \mathrm{C}\right)$, degrees Celsius; $\mathrm{ft}^{3} / \mathrm{s}$, cubic feet per second; $\mu \mathrm{S} / \mathrm{cm}$, microsiemens per centimeter; mg/L, milligrams per liter; Amn+org, ammonia plus organic; $\mathrm{NO}_{2}+\mathrm{NO}_{3}$, nitrite plus nitrate; --, insufficient number of uncensored data for statistical calculation]

\section{STATION NUMBER: 12509696 STATION NAME: SPRING CREEK AT HANKS ROAD NEAR PROSSER, WASHINGTON LATITUDE/LONGITUDE: 4616221194417}

Descriptive statistics

\begin{tabular}{|c|c|c|}
\hline $\begin{array}{l}\text { Para- } \\
\text { meter } \\
\text { code }\end{array}$ & $\begin{array}{l}\text { Water-quality } \\
\text { constituent }\end{array}$ & Units \\
\hline 00010 & Water temperature & (degrees C) \\
\hline 00061 & Discharge, inst. & $\left(\mathrm{ft}^{3} / \mathrm{s}\right)$ \\
\hline 00095 & Specific conductance & $\left(\mu \mathrm{S} / \mathrm{cm}\right.$ at $\left.25^{\circ} \mathrm{C}\right)$ \\
\hline 00300 & Oxygen, dissolved & $(\mathrm{mg} / \mathrm{L})$ \\
\hline 00400 & $\mathrm{pH}$, field & (standard unit) \\
\hline 00608 & Nitrogen ammonia & $(\mathrm{mg} / \mathrm{L}$ as $\mathrm{N})$ \\
\hline 00613 & Nitrogen, nitrite & $(\mathrm{mg} / \mathrm{L}$ as $\mathrm{N})$ \\
\hline 00623 & Amn+org, filtered & $(\mathrm{mg} / \mathrm{L}$ as $\mathrm{N})$ \\
\hline 00625 & Amn+org, unfiltered & $(\mathrm{mg} / \mathrm{L}$ as $\mathrm{N})$ \\
\hline 00631 & $\mathrm{NO}_{2}+\mathrm{NO}_{3}$, filtered & $(\mathrm{mg} / \mathrm{L}$ as $\mathrm{N})$ \\
\hline 00665 & Phosphorus, total & $(\mathrm{mg} / \mathrm{L}$ as $\mathrm{P})$ \\
\hline 00666 & Phosphorus, filtered & (mg/L as $\mathrm{P}$ ) \\
\hline 00671 & Phosphorus, ortho & $(\mathrm{mg} / \mathrm{L}$ as $\mathrm{P})$ \\
\hline 80154 & Suspended sediment & $(\mathrm{mg} / \mathrm{L})$ \\
\hline
\end{tabular}

Percent of samples in which values were less than or equal to those shown

\begin{tabular}{lll} 
Sample & Maxi- \\
size & mum & $\begin{array}{l}\text { Mini- } \\
\text { mum }\end{array} \quad$ Mean \\
\hline
\end{tabular}

\begin{tabular}{ccccc}
\hline & & & & \\
$95 \%$ & $75 \%$ & $50 \%$ & $25 \%$ & $5 \%$ \\
\hline & & & & \\
22.5 & 18.8 & 16.2 & 10.9 & 7.5 \\
11.2 & 8.2 & 5.8 & 1.2 & 0.15 \\
670 & 642.5 & 229 & 184 & 156 \\
13.2 & 10.9 & 9.2 & 7.7 & 6.7 \\
8.4 & 8.0 & 7.8 & 7.8 & 7.6 \\
$* 0.07$ & $* 0.04$ & $* 0.02$ & $* 0.013$ & $* 0.008$ \\
$* 0.02$ & $* 0.01$ & $* 0.01$ & $* 0.008$ & $* 0.006$ \\
$* 0.3$ & $* 0.2$ & $* 0.2$ & $* 0.2$ & $* 0.1$ \\
$* 0.6$ & $* 0.5$ & $* 0.3$ & $* 0.3$ & $* 0.16$ \\
6.1 & 5.4 & 1.2 & 0.71 & 0.37 \\
0.26 & 0.12 & 0.09 & 0.06 & 0.05 \\
0.07 & 0.05 & 0.04 & 0.03 & 0.02 \\
0.13 & 0.06 & 0.04 & 0.03 & 0.02 \\
226 & 104 & 42 & 14 & 4
\end{tabular}

\section{STATION NUMBER: 12509698 STATION NAME: SPRING CREEK AT MCCREADIE ROAD NEAR PROSSER, WASHINGTON LATITUDE/LONGITUDE: 4615271194237}

Descriptive statistics

\begin{tabular}{|c|c|c|c|c|c|c|c|c|c|c|c|}
\hline $\begin{array}{l}\text { Para- } \\
\text { meter } \\
\text { code }\end{array}$ & $\begin{array}{l}\text { Water-quality } \\
\text { constituent }\end{array}$ & Units & $\begin{array}{l}\text { Sample } \\
\text { size }\end{array}$ & $\begin{array}{l}\text { Maxi- } \\
\text { mum }\end{array}$ & $\begin{array}{l}\text { Mini- } \\
\text { mum }\end{array}$ & Mean & $95 \%$ & $75 \%$ & $\begin{array}{l}\text { (Median) } \\
50 \%\end{array}$ & $25 \%$ & $5 \%$ \\
\hline 00010 & Water temperature & (degrees C) & 19 & 20.5 & 9.0 & 14.6 & 20.5 & 18.0 & 15.0 & 10.7 & 9.0 \\
\hline 00061 & Discharge, inst. & $\left(\mathrm{ft}^{3} / \mathrm{s}\right)$ & 19 & 19.0 & 2.2 & 10.8 & 18.8 & 15.8 & 11.8 & 4.2 & 2.2 \\
\hline 00095 & Specific conductance & $\left(\mu \mathrm{S} / \mathrm{cm}\right.$ at $\left.25^{\circ} \mathrm{C}\right)$ & 19 & 750 & 236 & 427 & 750 & 730 & 317 & 278 & 236 \\
\hline 00300 & Oxygen, dissolved & $(\mathrm{mg} / \mathrm{L})$ & 19 & 11.6 & 8.3 & 9.9 & 11.6 & 10.6 & 10.0 & 9.0 & 8.3 \\
\hline 00400 & $\mathrm{pH}$, field & (standard unit) & 19 & 8.4 & 7.9 & 8.1 & 8.4 & 8.2 & 8.1 & 8.0 & 7.9 \\
\hline 00608 & Nitrogen ammonia & $(\mathrm{mg} / \mathrm{L}$ as $\mathrm{N})$ & 19 & 0.08 & $<0.015$ & $0.02 *$ & ${ }^{*} 0.08$ & $* 0.03$ & $* 0.02$ & $* 0.009$ & $* 0.005$ \\
\hline 00613 & Nitrogen, nitrite & $(\mathrm{mg} / \mathrm{L}$ as $\mathrm{N})$ & 19 & 0.02 & $<0.01$ & -- & -- & -- & -- & -- & -- \\
\hline 00623 & Amn+org, filtered & $(\mathrm{mg} / \mathrm{L}$ as $\mathrm{N})$ & 19 & 0.3 & $<0.1$ & $0.2^{*}$ & $* 0.3$ & $* 0.2$ & $* 0.2$ & $* 0.2$ & $* 0.1$ \\
\hline 00625 & Amm+org, unfiltered & $(\mathrm{mg} / \mathrm{L}$ as $\mathrm{N})$ & 19 & 1.1 & $<0.2$ & $0.4^{*}$ & $* 1.1$ & $* 0.6$ & $* 0.3$ & $* 0.3$ & $* 0.1$ \\
\hline 00631 & $\mathrm{NO}_{2}+\mathrm{NO}_{3}$, filtered & $(\mathrm{mg} / \mathrm{L}$ as $\mathrm{N})$ & 19 & 6.9 & 0.94 & 3.0 & 6.9 & 5.4 & 2.0 & 1.3 & 0.94 \\
\hline 00665 & Phosphorus, total & $(\mathrm{mg} / \mathrm{L}$ as $\mathrm{P})$ & 19 & 0.54 & 0.03 & 0.15 & 0.54 & 0.20 & 0.11 & 0.07 & 0.03 \\
\hline 00666 & Phosphorus, filtered & $(\mathrm{mg} / \mathrm{L}$ as $\mathrm{P})$ & 19 & 0.11 & 0.02 & 0.05 & 0.11 & 0.06 & 0.04 & 0.03 & 0.02 \\
\hline 00671 & Phosphorus, ortho & $(\mathrm{mg} / \mathrm{L}$ as $\mathrm{P})$ & 19 & 0.16 & 0.03 & 0.06 & 0.16 & 0.07 & 0.05 & 0.04 & 0.03 \\
\hline 80154 & Suspended sediment & $(\mathrm{mg} / \mathrm{L})$ & 19 & 664 & 6 & 132 & 664 & 172 & 88 & 35 & 6 \\
\hline
\end{tabular}

* - Value is estimated by using a log-probability regression to predict the values of data below the detection limit (Helsel and Cohn, 1S 88). 


\section{SEASONAL DIFFERENCES IN SURFACE WATER}

Concentrations of ammonia, ammonia-plusorganic nitrogen, total phosphorus, and suspended sediment generally were Iargest April through September, which is during the irrigation season; whereas the largest concentrations of dissolved nitrate and dissolved phosphorus generally occurred during the non-irrigation season. Although concentrations of dissolved nitrate are Iarger during the non-irrigation season, the instantaneous daily load of total nitrogen is generally larger during the irrigation season, because discharge is greater (table 3). Insufficient data do not permit a model of nutrient or sediment loads, but daily nutrient and sediment loads can be estimated from instantaneous concentrations and daily streamflow (table 3).

\section{QUALITY ASSURANCE AND CONTROL}

Quality-assurance (QA) plans were developed and followed to ensure that sample collection and processing procedures did not contaminate samples. The field QA plan consisted of quality-control procedures including the calibration of field instruments with standard solutions of known and assured values, submittal of quality-control samples that were free of analytes (blanks), and participation by field personnel in the National Field Quality Assurance Plan, as described by Stanley (1998). The laboratory QA plan consisted of quality-control procedures including the calibration of instruments and analysis of standard and blank solutions, as described by Pritt and Raese (1995). Field blanks were used to assess the potential levels of sample contamination occurring during sample collection, processing, and shipping. Two field blanks were collected on the same day as regular sampling (in February 1998 and in July 1998) by passing inorganic blank water through all sampling equipment using identical collection, processing, preservation, transportation, and storage procedures as for regular samples.

Nitrate was detected at a concentration of $0.008 \mathrm{mg} / \mathrm{L}$ in the February 1998 field blank; ammonia was detected at a concentration of $0.007 \mathrm{mg} / \mathrm{L}$, orthophosphate was detected at a concentration of $0.003 \mathrm{mg} / \mathrm{L}$, and nitrite was detected at a concentration of $0.001 \mathrm{mg} / \mathrm{L}$ in the July 1998 field blank. The blanks were analyzed using a special low-level technique, but the normal laboratory reporting limits (LRL) for nitrate, orthophosphate, nitrite, and ammonia are 0.05 , $0.01,0.01$, and $0.015 \mathrm{mg} / \mathrm{L}$, respectively. The normal LRL for nitrate is more than 5 times the value of the detection in the blank, the LRL for orthophosphate is 3 times the value of the detection in the blank, th: LRL for nitrite is 10 times the value of the detection in the blank, and the LRL for ammonia is more than tvice the value of detection in the blank; thus there is little chance that the values for these constituents are biased.

\section{SUMMARY}

A gage was installed to measure streamflow in the Spring Creek Basin and samples were collerted at two sites to measure concentrations of nutrients and sediment from April 1997 to September 1998. Concentrations of nitrate in samples from Spring Creek at Hanks Road ranged from 0.37 to $6.2 \mathrm{mg} / \mathrm{L}$ and concentrations of nitrate in samples from Spring Creek at McCreadie Road ranged from 0.94 to $6.9 \mathrm{mg} / \mathrm{L}$. Concentrations of phosphorus species and susponded sediment, showing a pattern of larger median concentrations and wider ranges at McCreadie Road than at the Hanks Road site, ranged from 0.01 to $0.07 \mathrm{mg} / \mathrm{L}$ and 0.05 to $0.26 \mathrm{mg} / \mathrm{L}$ at the Hanks Road site and ranged from 0.02 to $0.11 \mathrm{mg} / \mathrm{L}$ and 0.03 to $0.54 \mathrm{mg} / \mathrm{L}$ at the McCreadie Road site for dissolved and total phosphorus, respectively. Concentrations of suspended sediment ranged from 4 to $226 \mathrm{mg} / \mathrm{L}$ at the Hanks Road site and 6 to $664 \mathrm{mg} / \mathrm{L}$ at the McCreadie Road site. 
Table 3. Nutrient and sediment loads from two sites in the Spring Creek Basin, April 1997 to September 19?8, Benton County, Washington

[mg/L, milligrams per liter; N, nitrogen; P, phosphorus; $t /$ day, tons per day; $<$, less than]

\begin{tabular}{|c|c|c|c|c|c|c|c|}
\hline Date & Time & $\begin{array}{l}\text { Dis- } \\
\text { charge } \\
\text { (cubic } \\
\text { feet } \\
\text { per } \\
\text { day) }\end{array}$ & $\begin{array}{l}\text { Nitro- } \\
\text { gen } \\
(\mathrm{mg} / \mathrm{L} \\
\text { as } \mathrm{N})\end{array}$ & $\begin{array}{l}\text { Phos- } \\
\text { phorus } \\
\text { (mg/L } \\
\text { as } \mathrm{P} \text { ) }\end{array}$ & $\begin{array}{l}\text { Nitro- } \\
\text { gen } \\
\text { (t/day } \\
\text { as } N)\end{array}$ & $\begin{array}{l}\text { Phos- } \\
\text { phorus } \\
\text { (t/day } \\
\text { as } \mathrm{P} \text { ) }\end{array}$ & $\begin{array}{l}\text { Sedi- } \\
\text { ment, } \\
\text { dis- } \\
\text { charge, } \\
\text { sus- } \\
\text { pended } \\
\text { (t/day) }\end{array}$ \\
\hline \multicolumn{8}{|c|}{ Spring Creek at Hanks Road near Prosser. Washington } \\
\hline \multicolumn{8}{|c|}{ April 1997} \\
\hline $23 \ldots$ & 1200 & 535,680 & 1.8 & 0.11 & 0.03 & 0.002 & 1.7 \\
\hline \multicolumn{8}{|l|}{ May } \\
\hline $23 \ldots$ & 1300 & 388,800 & 2.1 & 0.23 & 0.03 & 0.003 & 2.7 \\
\hline \multicolumn{8}{|l|}{ June } \\
\hline $16 \ldots$ & 1315 & 665,280 & 1.7 & 0.26 & 0.04 & 0.005 & 4.1 \\
\hline \multicolumn{8}{|l|}{ July } \\
\hline $16 \ldots$ & 1200 & 803,520 & 1.1 & 0.10 & 0.03 & 0.003 & 0.96 \\
\hline \multicolumn{8}{|l|}{ August } \\
\hline $20 \ldots$ & 1310 & 838,080 & 1.4 & 0.15 & 0.04 & 0.004 & 2.4 \\
\hline \multicolumn{8}{|c|}{ September } \\
\hline $24 \ldots$ & 1400 & 622,080 & $\mathrm{e}_{1.5}$ & 0.05 & ${ }^{\mathrm{e}} 0.03$ & $\mathrm{e}_{0.001}$ & $0.2=$ \\
\hline \multicolumn{8}{|c|}{ October } \\
\hline $22 \ldots$ & 1300 & 276,480 & $\mathrm{e}_{3.4}$ & 0.06 & ${ }^{\mathrm{e}} 0.03$ & $\mathrm{e}_{<}<0.001$ & 0.10 \\
\hline \multicolumn{8}{|c|}{ November } \\
\hline $25 \ldots$ & 1330 & 120,960 & 6.3 & 0.11 & 0.02 & $<0.001$ & 0.04 \\
\hline \multicolumn{8}{|c|}{ December } \\
\hline $18 \ldots$ & 1300 & 69,120 & 6.5 & 0.08 & 0.01 & $<0.001$ & 0.10 \\
\hline \multicolumn{8}{|c|}{ January 1998} \\
\hline $27 \ldots$ & 1245 & 33,696 & 6.4 & 0.05 & 0.01 & $<0.001$ & 0.00 \\
\hline \multicolumn{8}{|c|}{ February } \\
\hline $25 \ldots$ & 1245 & 34,560 & 6.0 & 0.07 & 0.006 & $<0.001$ & 0.15 \\
\hline \multicolumn{8}{|l|}{ March } \\
\hline $18 \ldots$ & 1230 & 12,960 & 5.6 & 0.06 & 0.002 & $<0.001$ & 0.01 \\
\hline \multicolumn{8}{|l|}{ April } \\
\hline $23 \ldots$ & 1330 & 35,420 & 1.3 & 0.07 & 0.01 & $<0.001$ & 0.80 \\
\hline \multicolumn{8}{|l|}{ May } \\
\hline $19 \ldots$ & 1240 & 639,360 & 0.92 & 0.09 & 0.02 & 0.002 & 2.2 \\
\hline \multicolumn{8}{|l|}{ June } \\
\hline $24 \ldots$ & 1300 & 466,560 & 1.3 & 0.09 & 0.02 & 0.001 & 0.67 \\
\hline
\end{tabular}


Table 3. Nutrient and sediment loads from two sites in the Spring Creek Basin, April 1997 to September 19?8, Benton County, Washington--Continued

\begin{tabular}{|c|c|c|c|c|c|c|c|}
\hline Date & Time & 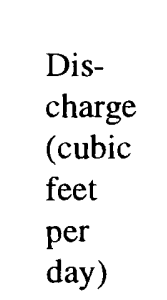 & $\begin{array}{l}\text { Nitro- } \\
\text { gen } \\
(\mathrm{mg} / \mathrm{L} \\
\text { as } \mathrm{N})\end{array}$ & $\begin{array}{l}\text { Phos- } \\
\text { phorus } \\
\text { (mg/L } \\
\text { as } \mathrm{P} \text { ) }\end{array}$ & $\begin{array}{l}\text { Nitro- } \\
\text { gen } \\
\text { (t/day } \\
\text { as } N \text { ) }\end{array}$ & $\begin{array}{l}\text { Phos- } \\
\text { phorus } \\
\text { (t/day } \\
\text { as P) }\end{array}$ & $\begin{array}{l}\text { Sedi- } \\
\text { ment, } \\
\text { dis- } \\
\text { charge, } \\
\text { sus- } \\
\text { pended } \\
\text { (t/day) }\end{array}$ \\
\hline \multicolumn{8}{|l|}{ July } \\
\hline $22 \ldots$ & 1255 & 673,920 & 0.65 & 0.12 & 0.01 & 0.003 & $0.5 \mathrm{~s}$ \\
\hline \multicolumn{8}{|l|}{ August } \\
\hline $20 \ldots$ & 1240 & 950,400 & 0.66 & 0.05 & 0.02 & 0.001 & 0.32 \\
\hline \multicolumn{8}{|c|}{ September } \\
\hline $08 \ldots$ & 1200 & 950,400 & 0.86 & 0.10 & 0.03 & 0.003 & 0.85 \\
\hline \multicolumn{8}{|c|}{ Spring Creek at McCreadie Road near Prosser, Washington } \\
\hline \multicolumn{8}{|c|}{ April 1997} \\
\hline $23 \ldots$ & 1030 & 950,400 & 2.8 & 0.22 & 0.08 & 0.007 & 5.8 \\
\hline \multicolumn{8}{|l|}{ May } \\
\hline $23 \ldots$ & 1020 & $1,036,800$ & 2.7 & 0.23 & 0.09 & 0.007 & 10 \\
\hline \multicolumn{8}{|l|}{ June } \\
\hline $16 \ldots$ & 1100 & 950,400 & 3.2 & 0.54 & 0.10 & 0.016 & 6.9 \\
\hline \multicolumn{8}{|l|}{ July } \\
\hline $16 \ldots$ & 0950 & $1,036,800$ & 2.3 & 0.10 & 0.07 & 0.003 & 2.9 \\
\hline \multicolumn{8}{|l|}{ August } \\
\hline $20 \ldots$ & 1000 & $1,209,600$ & 2.0 & 0.08 & 0.08 & 0.003 & 2.7 \\
\hline \multicolumn{8}{|c|}{ September } \\
\hline $24 \ldots$ & 1205 & $1,382,400$ & 2.5 & 0.11 & 0.11 & 0.005 & 3.9 \\
\hline \multicolumn{8}{|l|}{ October } \\
\hline $22 \ldots$ & 1100 & 656,400 & $\mathrm{e}_{4.4}$ & 0.07 & ${ }^{\mathrm{e}} 0.09$ & 0.001 & 0.66 \\
\hline \multicolumn{8}{|c|}{ November } \\
\hline $25 \ldots$ & 1050 & 362,880 & 7.1 & 0.04 & 0.08 & $<0.001$ & $0.0 S$ \\
\hline \multicolumn{8}{|c|}{ December } \\
\hline $18 \ldots$ & 1150 & 285,120 & 7.0 & 0.08 & 0.06 & $<0.001$ & 0.05 \\
\hline \multicolumn{8}{|c|}{ January 1998} \\
\hline $27 \ldots$ & 1030 & 241,920 & 6.7 & 0.04 & 0.05 & $<0.001$ & 0.05 \\
\hline \multicolumn{8}{|c|}{ February } \\
\hline $25 \ldots$ & 1030 & 224,640 & 5.7 & 0.04 & 0.04 & $<0.001$ & 0.62 \\
\hline \multicolumn{8}{|l|}{ March } \\
\hline $18 \ldots$ & 1020 & 190,080 & 6.3 & 0.03 & 0.04 & $<0.001$ & 0.21 \\
\hline
\end{tabular}


Table 3. Nutrient and sediment loads from two sites in the Spring Creek Basin, April 1997 to September 1998, Benton County, Washington--Continued

\begin{tabular}{|c|c|c|c|c|c|c|c|}
\hline Date & Time & $\begin{array}{l}\begin{array}{l}\text { Dis- } \\
\text { charge } \\
\text { (cubic }\end{array} \\
\text { feet } \\
\text { per } \\
\text { day) }\end{array}$ & $\begin{array}{l}\text { Nitro- } \\
\text { gen } \\
\text { (mg/L } \\
\text { as } \mathrm{N} \text { ) }\end{array}$ & $\begin{array}{l}\text { Phos- } \\
\text { phorus } \\
(\mathrm{mg} / \mathrm{L} \\
\text { as } \mathrm{P})\end{array}$ & $\begin{array}{l}\text { Nitro- } \\
\text { gen } \\
(\mathrm{t} / \mathrm{day} \\
\text { as } N)\end{array}$ & $\begin{array}{l}\text { Phos- } \\
\text { phorus } \\
\text { (t/day } \\
\text { as P) }\end{array}$ & $\begin{array}{l}\text { Sedi- } \\
\text { ment, } \\
\text { dis- } \\
\text { charge, } \\
\text { sus- } \\
\text { pended } \\
\text { (t/day) }\end{array}$ \\
\hline \multicolumn{8}{|l|}{ April } \\
\hline $23 \ldots$ & 1030 & 838,080 & 2.0 & 0.10 & 0.05 & 0.003 & 1.9 \\
\hline \multicolumn{8}{|l|}{ May } \\
\hline $19 \ldots$ & 1015 & $1,382,400$ & 1.6 & 0.11 & 0.07 & 0.005 & 7.3 \\
\hline \multicolumn{8}{|l|}{ June } \\
\hline $01 \ldots$ & 1200 & $1,468,800$ & 1.4 & 0.20 & 0.06 & 0.009 & 7.2 \\
\hline $24 \ldots$ & 1100 & $1,123,200$ & 1.9 & 0.11 & 0.07 & 0.004 & 2.7 \\
\hline \multicolumn{8}{|l|}{ July } \\
\hline $22 \ldots$ & 1040 & $1,296,000$ & 2.0 & 0.38 & 0.08 & 0.015 & 28 \\
\hline \multicolumn{8}{|l|}{ August } \\
\hline $20 \ldots$ & 1020 & $1,382,400$ & 2.0 & 0.15 & 0.09 & 0.006 & 3.3 \\
\hline \multicolumn{8}{|c|}{ September } \\
\hline $08 \ldots$ & 1050 & $1,641,600$ & 1.8 & 0.20 & 0.09 & 0.010 & 6.6 \\
\hline
\end{tabular}

e Total nitrogen (the sum of $\mathrm{NO}_{2}+\mathrm{NO}_{3}$ and ammonia plus organic) is estimated using the laboratory reporting limit for the ammonia plus organic value for all samples with concentrations of ammonia plus organic nitrogen that are less than the laboratory reporting limit. The calculated loads are also reported as an estimate. 


\section{SELECTED REFERENCES}

Daly, Pat, 1998, Spring Creek water quality and GIS mapping project: Benton Conservation District, Centennial Clean Water Program, 101 p.

Edwards, T.K., and Glysson, G.D., 1988, Field methods for measurement of fluvial sediment: U.S. Geological Survey Open-File Report 86-531, 118 p.

Fishman, Marvin J., 1993, Methods of analysis by the U.S. Geological Survey National Water Quality Laboratory-determination of inorganic and organic constituents in water and fluvial sediments: U.S. Geological Survey Open-File Report 93-125, 217 p.

Guy, Harold P., 1969, Laboratory theory and methods for sediment analysis: Techniques of Water-Resources Investigations of the United States Geological Survey, Book 5, Chapter $\mathrm{C} 1,58 \mathrm{p}$.

Helsel, Dennis R., and Cohn, Timothy A., 1988, Estimation of descriptive statistics for multiply censored water quality data: Water Resources Research, v. 24, p. $1,997-2,004$.

Horowitz, A.J., Demas, C.R., Fitzgerald, K.K., Miller, T.L., and Rickert, D.A., 1994, U.S. Geological Survey protocol for the collection and processing of surface-water samples for the subsequent determination of inorganic constituents in filtered water: U.S. Geological Survey Open-File Report 94-539, 57 p.
Patton, C.J., and Truitt, E.P., 1992, Methods of anal'sis by the U.S. Geological Survey National Water Quality Laboratory--determination of total phosphorus by a Kjeldahl digestion method and an automated colorimetric finish that includes dialysis: U.S. Geological Survey Open-File Report 92-146, 39 p.

Pritt, J.W., and Raese, J.W., 1995, Quality assurance/quality control manual--National Water Quality Laboratory: U.S. Geological Survey Open-File Report 95-443, 35 p.

Rinella, J.F., McKenzie, S.W., and Fuhrer, G.J., 19S2, Surface-water quality assessment of the Yakima River Basin, Washington--analysis of available water-quality data through 1985 water year: U.S. Geological Survey Open-File Report 91-453, 244 p., 1 pl.

Stanley, Daniel L., 1998, Summary of the U.S. Geo'ngical Survey National Field Quality Assurance Program from 1979 through 1997: U.S. Geological Survey Open-File Report 98-392, 11 p.

Wiggins, W.D., Ruppert, G.P., Smith, R.R., Reed, L.L., and Courts, M.L., 1998, Water resources data Washington water year 1997: U.S. Geological Survey Water-Data Report WA-97-1, 528 p.

Wiggins, W.D., Ruppert, G.P., Smith, R.R., Hubbar I, L.E., and Courts, M.L., 1999, Water resources data V'ashington water year 1998: U.S. Geological Survey WaterData Report WA-98-1, 508 p. 